\title{
Service User Involvement: Enriching the Learning Experience
}

\section{Jill Leckey ${ }^{1}$ Phil Walters ${ }^{2}$, Barrie Holt ${ }^{2}$}

\author{
${ }^{1}$ University of Huddersfield \\ ${ }^{2}$ South West Yorkshire Mental Health NHS Trust
}




\title{
Service User Involvement: Enriching the Learning Experience
}

\author{
Jill Leckey, Phil Walters, Barrie Holt:
}

Key Words: Service User, Collaboration, Involvement, Education Services, Complexity, Support.

\begin{abstract}
:
Recent policy initiatives e.g. The Health Care Commission (2005) have promoted the idea that services should be developed in collaboration with the service users, to meet the needs of those using them, to improve health and health care for everyone. Sainsbury Centre for Mental Health (2006) further supported this and pointed out that some aspects of the mental health services should take direction from the service users. Based on the principle that if service delivery is characterised by an ethos of collaboration, then such collaboration must also be the bedrock of mental health education (Tew et al 2004). This paper purports that by virtue of the service user's experience of mental distress and service provision, they offer valuable knowledge and expertise to enrich the Mental Health nursing students learning experience collaboratively. This paper considers a historical perspective of the issues, shares some positive examples of good practice around involvement and inclusion considering the conflicting tensions that became complementary and how this helped, in developing an enriching learning experience.
\end{abstract}

\section{Reviewing the Past}

The South West Yorkshire Mental Health Trust (SWYMHT) are applying for Foundation Trust status as it will give them the freedom to run their own affairs while remaining fully within the NHS. This means they will be accountable to local people and to an independent regulator, called Monitor. Being a Foundation Trust should help them to be more responsive to local needs and to work with their partners to improve and develop services (SWYMHT 2007).

The Trust has always involved service users and their carers in influencing how services are planned and delivered. Becoming a Foundation Trust will helps to formalise this way of working and will give all local people an even greater say in how services develop in the future (SWYMHT 2007).

Many mental health trusts are moving towards foundation status that should lead to a more democratic approach to delivering mental health care and as indicated a greater community ownership of mental health services. This is being mirrored within Higher Education with greater involvement of service users with the educational process (Breeze 2005). Although service user involvement has been high on the agenda and was incorporated within the 1982 syllabus of training (English National Board 1982), involvement has been cumbersome, tokenistic and slow. Harrison (2002) raises the issue that service user involvement can no longer be seen as a burdensome addition to health and needs to become part of everyday practice. The Department of Health further support this development with their 10 year guide that stresses service user involvement in the 
development of services and the educational process (Department of Health 2005).

Service user involvement again reached the top of the agenda within Mental Health policy, with a shift away from professional dominance to a partnership which aims to respond to the needs of the individuals (Repper \& Perkins 2001). This notion purports that individuals are the experts and the health professional are the supporters. This is contrary to the traditional medical model in psychiatry which sets up the doctor/nurse and service user relationship as the "professional" knows best and the "service user" as a passive recipient of care/treatment. This relationship in its purest form is disabling and very disempowering (Foucault 1980). The old asylum system itself, rather than being a place of care and treatment was a deliberate act of social exclusion which suited the political and economic climate at the time. This system created individuals who could not function due to institutionalisation and found it difficult to integrate back into society due to the stigma associated with mental illness (Department of Health 2006).

Inevitably the backlash came in the 1960's, both from professionals in terms of the anti-psychiatry movement, and from service users in the form of the very damming survivor movement working in collaboration. The power at this point was still with the establishment and most of the protests were undermining the powers of the medical profession and society's faith in science, having all the answers (Foucault 1980).

Ron Coleman (1998) put forward a very compelling argument which often makes uncomfortable reading, but challenges the very core of the power imbalance in our society. He (1998) argues that the ruling classes decided what was and what was not acceptable in society, it was they who defined madness even in individual cases, hence when one of their own was afflicted; it was a case of eccentricity. It was in the main upper middle class males who became psychiatrists and it is for this reason that psychiatry is still sexist, racist, homophobic and anti-poor (Colman 1998).

Mind (2008) suggest that the user/survivor movement is characteristically diverse and almost impossible to define. Many mental health service users/ survivors express their anger and distress at having been defined by the psychiatric system and by the wider society. Many have experienced the stigma of a diagnostic label, like 'schizophrenic' 'manic' or 'neurotic' and the hurt of being labelled 'loony', 'nutter' or 'mental' by society at large. It is perhaps inevitable then that language has a particular relevance and importance for many users/survivors.

www.mind.org.uk/information/factsheets (2008)

This starts to illustrate that any concessions to the involvement and inclusion of those who suffer mental distress have been fought hard and long. A significant breakthrough was a land mark piece of research carried out by Romme and Esher (1993). They discovered that two thirds of the people they contacted who heard voices were not in touch with mental health services. These people were often coping better than those in touch with services and had developed their own coping mechanisms.

The hearing voices network is an example that followed this work, started to establish the idea that people that suffer mental distress are the experts in their own care. The mental health services could have learned a lot from 
their early involvement. The influence of this work and the work of the networks can now be seen in the general shift towards more psycho-social approaches to mental health, with a recovery focus and the development the critical psychiatry movement and complimentary therapy approaches to mental health care (Barker 2004). This move away from mental health services dominated by the medical model to a broader more holistic approach has been a long time coming for many. This has gained impetus with the recovery model and strengths based care leading the way in the development of services and the education of future nurses (Shepherd, Boardman \& Slade 2008)

The broader political landscape has also changed quite radically to embrace a more socially inclusive model for mental health care, which promotes the idea that people who have suffered mental distress have a lot to contribute. This leads the development of policy/legislation which directly supports and encourages a more inclusive approach. This includes section 11 of the Health and Social Care Act 2001, Commissioning a Service user Led NHS (2005) and the White Paper 'Our Health, Our Care, Our Say' (Department of Health 2005).

The guiding principles to promote the process of service user involvement within nursing and the educational process have been the Disability Discrimination Act (1995), Health \& Social Care Act (2001), Employment Act (2002), Human Rights Act (1998) \& Equal Opportunities Act (1996) \& Service user \& Public Involvement in Health (2004). Masters et al (2002) emphases that service users and carers should actively engage in partnerships with education professionals in all aspects of the curriculum to add richness and meaning to the process. A stark realisation, but where do we begin? What are the issues?

Students within the University of Huddersfield embarking on their mental health nursing careers spend $50 \%$ of their total hours in the clinical areas working with service users and carers and $50 \%$ within the educational context.

One of the issues that this raised was that students were only seeing one side of the picture working with service users engaged currently with services and not those beyond. To involve service users within the educational context meant that the students were working along side individuals who were not necessarily currently utilising services so it added another perspective to the educational process (Forrest et al 2000).

\section{The Service User's Perspective of the Process}

It is important from a service user's perspective that service user involvement in the work of the University of Huddersfield and the South West Yorkshire Mental Health Trust is effective. A personal observation on meeting other service users that is interesting to note, is that the range of complaints they have made is in relation to the poor treatment they perceive to have been given by the providers in the mental health services. This can largely be attributed to misunderstandings. If we are to get the best service delivery and treatments possible then we need to look much more carefully at how we interact at the point of delivery both from a service user perspective and professional delivery and interaction. 
For this to happen, education, service users and health professionals need to work more closely together. The Government and NHS trusts require us to develop a much more user-friendly service. How can we work towards that ideal, and how can we engage service users in the process? There are a number of fundamental issues that need to be addressed if we are to achieve this for example: in communication and education.

A common factor within a complaint is the lack of understanding of the difficulties and sensitivities the service user feels during a mental illness. The loss of self- respect, confidence and stigma attached to mental illness, all contribute to a negative perception of their treatment. Many service users feel that they are not important in the process of service delivery. This has a negative effect on service users and is not a pattern which aids recovery. Why do people feel this? What are the circumstances, which lead to this poor experience? These are complex and difficult questions to answer. The NHS trust has a large and complex organisation to manage with a wide demographic character, but the fundamental purpose is to deliver a service to fulfil the needs of the service user.

Mahatma Gandhi (1910) raised the point that we exist because of the needs of others. This suggests that if we do not meet the needs of our service users and when we do not provide the service user needs, there is no point in our existence. It is not as easy as it sounds.

This may seem obvious, yet we still have difficulties in communication between the service user and the service provider, particularly, during this time of major changes in service reorganisation. In discussions with other service users some of them complain of a lack of basic information about their mental health issues, others, the lack of consultation when services are changed, and people transferred between locations and practitioners.

If the NHS is to increase its quality of delivery and service user care, it must involve the service user in the process of service design and evaluation. A difficulty with this is that of recruiting service users to be involved. I think there are many who are only too happy to be involved, with high levels of skill, but do not know how to go about getting involved. There is also the issue that whilst service users are willing to be involved, staff are unsure about what level of involvement and how to utilise their involvement effectively (Social Care Institute of Excellence 2007).

We have established that there are conflicts of interest between service users and service providers, where they have their own agenda's, which do not always coincide. This places the service user at a disadvantage in influencing the direction and quality of service provision. The Department of Health (2004) postulate that in general, health professionals are positive about service user involvement, seeing it as a rewarding process for themselves as well as for the service user. These positive attitudes are, however, constrained by wider professional attitudes, ownership and expectations.

The Department of Health further develop this by identifying that different professionals have different understanding and attitudes of involvement. Nurses, for example, are supportive of the principles of involvement but their willingness to promote involvement can conflict with a professional ethic of protecting the service user from negative or exploitative 
experiences. The potential for conflict is evident between the practice of service user involvement and professional guidelines which may not be compatible with each other and therefore cause confusion and uncertainty in relation to implementation (Department of Health 2004).

Social Care Institute of Excellence (2007) clearly identify that there is a very strong need for information which informs the service user how they can be involved and at the same time is welcoming and supportive. It must explain what the Higher Education Institute and the NHS needs from that involvement and what level of involvement is open to them, how they will be supported, with details of responsibilities, and issues of payment and its effects on benefits the service user may be receiving. There are training programmes in place within West Yorkshire similar to the induction training given to NHS staff for service users who want to be involved (Tew et al 2004).

Also, a culture of trust between the service provider and the service user needs to be further developed, so that involvement means involvement, and it is not seen on either side as tokenism. It must be clear within the collaborative process that all are working for a common purpose (Breeze et al 2005). For this to happen groups need to be informed and co-operative, and develop understanding that staff have expertise in delivery, and service users have expertise in the illness, and that one is of no use without the other, and, that we all have a wide variety of skills that we can apply in improving the service.

To achieve improvements in service delivery a greater understanding of the issues that affect service users who chose to be involved needs to be strategically and consistently developed and applied (Sainsbury Centre for Mental Health 2006). At the moment it seems that the recruitment process for service user involvement is very much ad hoc, and fails in getting enough people to be involved through the lack of a clear strategy and poorly developed recruitment and support structures. It is not acceptable to expect someone who may have anxiety and feelings of worthlessness to come into the system to be asked to be involved, and then be left without a real understanding of their role and responsibilities, as this may have the effect of maintaining a sense of isolation. This perceived isolation can in itself can have a negative effect, and not be supportive and beneficial in that individual's recovery process.

As a service user of such services, clearly defined roles and levels of involvement are a necessity so that service users can decide where and how deeply they wish to be involved. Open forums are needed where issues of service user complaints or observations can be aired, and information about resolution of issues can be cascaded. A network is needed across the services to enable continuous dialogue between service users and service providers so that suggestions for improving services and environments can be discussed and implemented quickly ensuring that the service can develop high levels of service user care and support.

All this requires changes from service providers and service users alike. As a community we need to work hard with a common voice to bring a more enlightened attitude towards mental health and attempt to reduce the prejudice and stigma (Sainsbury Centre for Mental Health 2006). To do this we need to be involved, as expert service users, in the process of 
education, for staff, service users, and the public at large, so that a lot of the issues are addressed which have such a damaging effect on service users. We have to be aware of the pressures on staff, and as service users support them in the changes which will come from greater involvement.

We, as a mental health community, need to be much more involved in the education process and demonstrate that we have the skills and capacity to make quality contributions to the community and we need to start this at an early age.

Mental health education should start at an early stage with speakers from the mental health community (professionals and service users) talking with youngsters in schools about the causes of stress and bullying and how to deal with it. We need to promote an ethos, which values all members as being able to make a contribution towards its better development. This process is currently being rolled out with an initiative which sees staff and service users from the university running workshops for schools, but only to the schools that request this input. This is an area that could be developed further as the present system leaves the choice to individual staff. A more inclusive system would be beneficial which, incorporates mental health issues within the curriculum rather than an add on, examples of this can be found at; (www.geneticfutures.com/cracked/info/sheet6.asp. 2007)

In the education and training sectors, it is of paramount importance to identify those at risk of mental illness and provide the right sort of support so that they can still make a contribution to the economy whilst keeping their social networks, relations and status in society. If we as experts, having experience of the damaging effects of mental illness do nothing to change the status quo, who will? In general, health professionals are less likely to encourage service user involvement in decision-making (Department of Health 2004) if this conflicts with their own perceptions of what services should be provided.

The education and training of health professionals must have contributions from service users so that a better understanding of the issues develops and that more appropriate interventions become the accepted practice. This should always be an ongoing process. Likewise service user education should also be an important part of their involvement in work for the services. Research and service evaluation is always needed to address the quality and appropriateness of interventions. For this to be possible, the credibility of their findings needs to be supported by high quality investigations supported by evidence and good reporting. Therefore an important skill that needs to be developed is research methodology and should be part of the training programme for service user involvement.

This issue is currently being addressed within West Yorkshire and patient and public initiatives are being developed, for example West Yorkshire Universities service user and carer involvement have been developed for service user training within higher education. Other initiatives have been developed within the statutory and voluntary sectors that have programmes to support the service user.

Vitally important parts of service user involvement training are the issues of security, confidentiality and diversity. This has to address the capacity of the service user to understand and apply the policy of the trust and will 
involve an understanding of the issues raised in health and safety as well as disability discrimination and social inclusion (Sainsbury Centre for Mental Health 2006). It is therefore important that we create a structure, which is tiered to allow all levels of capacity and willingness of involvement to be possible (Breeze et al 2005). Only then will we see the dream of a real health service that can truly say it serves the needs of the community.

The stark reality is that the majority of individuals utilise a variety of services, although many not beyond complimentary approaches or primary care services (Sainsbury Centre for Mental Health 2006). So what makes a service user a service user? The answer to this question is multifaceted as it seems it is dependent upon use of services and diagnosis. This highlights the need to challenge mental health perceptions from both an external perspective and from within oneself (Holt 2005).

It is therefore useful to regard mental health as a continuum, on which all mental health states feature, it is not something that happens to other people, it can happen to anyone (Repper \& Perkins 2001). Deegan (1992) identifies a central attitudinal barrier that people with mental health problems can be ignored which is still evident today 15 years on, as individuals begin the journey along the Cycle of Disempowerment and Despair with the ethos that the professional 'knows best'( Deegan (1992).

Forrest et al (2000) noted that service user perceptions of their needs together with the level of support, that their need differs from the 'professionals' perspective. Repper and Perkins (2001) highlighted the need for mental health care workers to recognise that individuals are able to make their own choices and decisions. Mental Health workers should focus on the supportive role in relation to the service user not the other way round.

\section{The Story so Far}

The Sainsbury Centre for Mental Health (2006) recommends the involvement of service users in all aspects of mental health care, ranging from practice to education. The involvement of service users in curriculum design and delivery promotes an active rather than passive role for service user $\mathrm{s}$ which enhances and enriches the learning experience. Hanson \& Mitchell (2001) identified that the perception that service users are dependant and inadequate is challenged with their involvement within the educational process and service delivery.

The diverse nature of service user involvement is all too evident within the current systems. Anthony \& Crawford (2000) acknowledge this highlighting that the underpinning assumptions of nurses and users in relation to the characteristics of mental health problems are sometimes at odds. This results in a tension between viewing service users as rational thinking individuals who have a right to be involved, to the extreme, they are a danger to themselves and others and therefore not capable of rational thought (Rush 2004).

Lessons from history identify that these tensions do not resolve themselves and that a clear dialogue is needed. To some extent bio psychosocial interventions promote dealing with issues thereby promoting a recovery based service (Repper \& Perkins 2003 ) supporting the perception that the 
service user is the expert with the health care professional taking the role of the supporter. Although the culture is slowly changing it will take time, education and a change in the strategic planning and service organisation to implement strengths based service delivery, with the service user and their carers taking the lead in identifying their needs and informing the assessment process towards care pathways and packages, has to be the way forward.

The Chief Nursing Officer's Review (2006) in which critical feedback from service users about the impact of the nurse's behaviour and attitudes on the recovery process further supports and adds impetus to the need for a cultural change within services. This document clearly outlines the core competencies and capabilities, including knowledge and performance criteria, essential for mental health nurses at the point of registration. The notion is that additional competencies and capabilities will be incorporated at local level in collaboration with all concerned to meet the needs of the service users and careers within the geographical area of the local trust.

So far within the university successful steps are being made to involve service users and carers within the educational process to help achieve these competencies and capabilities identified within the Chief Nursing Officer's Review (2006). Due to the complexity of the issues, individuals experience different levels of support. Likewise there are different levels of involvement (Breeze et al 2005). These range from service user involvement to full inclusion \& widening participation where a list of Service users and their level of availability is held and access and input can be negotiated. This data base which identifies individuals, their contact details and their area of interest, is continually updated and developed by the service user project workers. Alongside this runs an accredited course to provide, education, training and support to further develop service user participation within the educational process lead by a dedicated lecturer within the university.

The university works alongside the Patient Advice and Liaison Service (PALS) across the South West Yorkshire Mental Health Trust: These teams lead on service user care issues and involvement and the university staff are part of these teams and engage in the planning of local projects involving the service users, carers and students.

There are many projects of involvement/inclusion within the planning stages for Mental Health Action Week, for example a mental health market, a concert, World Mental Health Day 2008 and a conference looking at the arts within mental health and the power of creativity. The Division of Mental Health has invited service users to participate within curriculum development. At a recent course validation, service users were involved within the consultation process in a variety of activities; these included module readers, involvement in the planning group and giving input and feedback on the skill competency requirements, giving feedback on wording and appropriateness of the documentation. Currently we are rewriting our curriculum for validation in partnership with service providers and service users. The process undertaken was not always easy as views differed, and at times were in conflict, but through open and honest dialogue these issues were usually resolved and the differing perceptions added a richness to the development of the course in terms of both process and content. 
There are currently many other levels of involvement (Breeze et al 2005) which include service user involvement on the Department of Health's skills for health. Some are involved in the provision of live case histories, but cognisance has to be given in relation to reliving the event and personal experience. Because of the nature of their issues it is paramount to ensure that an adequate level of support is provided to the individual. For example, to achieve this, team teaching is frequently utilised. Service users are also directly involved in the development of teaching materials and methods. They are also engaged in group and project work with both staff and students. Another aspect of service user involvement is in the planning of collaborative events such as World Mental Health Day, Conferences, lectures and inter-professional activities.

The development of a collaborative better mental health group between University staff, NHS staff, service users and carers has enabled an open dialogue, where differing opinions, approaches, values and experience can be aired in an environment that promotes an ethos of equality. This group was formed not by any directive but by a group of like minded people who were involved in planning events to celebrate mental health. This has cascaded into involvement in the many aspects of the educational process and service development. Further development is currently in the planning stages for greater inclusion and involvement.

A number of service users are employed as guest lecturers within the University. Likewise, staff are involved and have formed working alliances with service user led groups like Magpie, who campaign to ensure equity and have undertaken sterling work in relation to equal opportunities for service users with mental health needs and PALS services working across the South West Yorkshire Trust dealing with issues of service user concerns, develop networks, and support mechanisms that promote inclusion. Links were also made with the Positive Messages group, which was set up to disseminate understanding of mental health issues in the community and strived for inclusion and has developed materials and news letters distributed to the wider public demystifying mental health. Vocational Enterprises was an invaluable source of support helping to re-establish roles. The Pathways Day Centre, which provides support and a social network, also developed Frontline, a magazine designed to promote service users' views and issues, and Headstrong, an internet link that addresses aspects of mental health promotion. Artists in Mind has mentors who help and support individuals producing works that have been exhibited in local galleries for all to enjoy, and finally, workforce development promoting inclusion and social enterprise.

With all this involvement comes responsibility and due to the complexity of life and its effects, service users sometimes find it difficult to think clearly in the abstract rather than the concrete, therefore a support network is essential. To further enhance this, a handbook and toolkit are currently being developed by the service users to support the service users. A number of service users have commented on approaches to inclusion and identify that:

"It is not what is said, It is the way that it is said".....

Issues of communication, cascading information and education need further attention, to further promote a meaningful dialogue and equal 
integration. Department of Health (2004) identified that health professionals are aware of the importance of good communication with service user $s$ but do not always think through what this means in practice.

As with any journey, we cannot decide on the best route or mode of transport until we have talked at length with the person we are going with, and have agreed some destination. Likewise with service user involvement many policies and guidelines are issued and local trusts implement their own protocols, but careful thought needs to be given to the destination, to make it long term rather than a day trip, it needs people with 'vision'. With help the many skills and talents of the service users can be enlivened and incorporated to enhance both the service and educational needs of all those involved within care delivery. One area that needs to be developed further is that of carer involvement to ensure a more cohesive understanding adding richness to the educational process.

\section{In Conclusion}

Service user and provider involvement is actively sought within the division of mental health in the delivery, development and direction of the education process. Due to the complexity of the issues and the needs of the service users this occurs at various levels, as not all service users of the services or providers have the confidence or desire to stand up in front of students to talk about themselves or their lives. One of the main reasons, being the fear of reliving the events which have caused them distress.

The educational pathway has been developed, and planned by, service users of the services, students, carers, local trusts and the mental health team. Other departments within the university have participated in the development of some of the educational activities. Diverse service user groups have also been involved in the development of various activities and teaching sessions and this has promoted partnerships working across the curriculum. Training packages and support mechanisms are currently being considered and developed to support the individual's roles within the educational process.

Achieving meaningful involvement of service users and carers within the educational process is a journey of discovery. There is no 'one size fits all' model that will work for all programmes in all localities, what works is the commitment, dialogue and hard work of all involved (Tew et al 2004).

As identified, the process undertaken was not always easy, as views did differ and at times there was conflict, but through open and honest dialogue these issues were usually resolved and as stated previously the differing perceptions added richness to the development of the course in terms of both process and content. A thought to ponder....... one issue that arises time and time again is that papers are published on service user involvement in services, but there seems to be little or no real implementation, things appear to reach a dead end. The only thing that remains is the name on the paper.

At the moment implementation is undertaken by different individuals and organisations utilising various approaches, as the culture changes. When looking at the literature there are many papers on the policies and ways of involving service user $s$ but little or no work done on the effectiveness. 
There needs to be a lot more effort made to understand the process needed for effective involvement but where issues have a negative outcome these should be shared so that involvement is effective, cost efficient and a positive experience. It maybe a more cohesive approach will be adopted which clearly identifies the role and function of all those involved within care delivery.

Lessons have been learned through the Better Mental Health group as individuals now work as one for one purpose (Tew et al 2004) and have a common goal to celebrate mental health. We could all learn from this evolving approach. 


\section{References}

Anthony, P. \& Crawford, P. 2000. Service User Involvement in Care Planning: The Mental Health Nurse's Perspective. Journal and Psychiatric and Mental Health Nursing, 2000, 7, 425-434.

Barker, P, J. 2004. Assessment in Psychiatric and Mental Health Nursing: In Search of the Whole Person. Nelson Thornes: Cheltenham.

Birchall, J. \& Simmons, R. 2004. User Power: The Participation of Users in Public Services. National Consumer Council. London.

Breeze, J. 2005. Power Shift Promotes Partnership. Mental Health Nursing. May 2005; 25(3): 4-7.

Carers (Equal Opportunities) Act 2004: Elizabeth II. Chapter 15. Reprinted March 2005.

Coleman, Ron. 1998. Politics of The Mad House, Handsell Publishing.

Deegan, P. 1992. The Independent Living Movement \& People with Psychiatric Disabilities: Taking Back Control Over our Own Lives'. Psychosocial Rehabilitation Journal 15, 5-19.

Department of Health., 2001. Health and Social Care Act. HMSO. London.

Department of Health. 2004. Service User \& Public Involvement. HMSO. London.

Department of Health. 2005. Our Health, Our Care, Our Say'.HSMO. London.

Department of Health. 2005. Consultation on Future Support for Service User \& Public Involvement in Health-The Findings. HMSO. London.

Department of Health. 2006. Best Practice Competencies \& Capabilities for Pre-registration Mental Health Nurses in England. The Chief Nursing Officer's review of Mental Health Nursing. HSMO. London.

Department of Health. 2006. From Values to Action: The Chief Nursing Officer's Review of Mental Health Nursing. HSMO.

Department of Health. 2006. Action on Stigma: Promoting Mental Health, Ending Discrimination at Work. Gov.uk/publications. London

Department of Health. 2006. National Institute for Mental Health in England: Key Skills for Key Staff: A Competency Guide for Primary Care and Community Services. Gov.uk/publications. London.

Disability Discrimination Act. 1995., Elizabeth II: Chapter 50. Corporate Author: Great Britain. Publisher: Stationery Office.

Employment Act. 2002. Elizabeth II: Chapter 22. Corporate Author: Great Britain. Publisher: Stationery Office. 
English National Board for Nursing. 1988. The Syllabus of Training 1982: Professional Register - Part 3 Registered Mental Nurse. Bocardo Press Limited. Oxford.

Foucault, M. 1980. Power/Knowledge Selected Interviews \& Other Writings. Harvester Press: Brighton. Sussex.

Forrest, S., Risk, I., Masters, H. \& Brown, N. 2000. Mental Health Service User Involvement in Nurse Education: Exploring the Issues.

Gandhi, M. 1910. Cited in Boyd, R.2006. The Value of Civility. Urban Studies Vol 43. Issue 5/6. p863-878, 16p.

Journal of Psychiatric \& Mental Health Nursing: 2000, 7, 51-57.

Hanson, B. \& Mitchell, D, P. 2001. Involving Mental Health Service Users in the Classroom: A course of Preparation. Nurse Education in Practice 2001. $1,120-126$.

Harrison, M., 2002. Perils of Engagement. Mental Health Today: September 2002: 28-30.

Health Care Commission. 2005. Engaging Service Users \& the Public: Have you Say: Health Care Commission. London.

Holt, B. 2005. Then and Now. Positive Messages News Letter: South West Yorkshire Mental Health Trust, January 2005.

Human Rights Act. 1998: Elizabeth II. Chapter 42 Reprinted May 2001 and September 2006. Corporate Author: Great Britain. Publisher: Stationery Office.

National Social Inclusion Programme CSIP 2006 Second Annual Report: National Social Inclusion Programme. CSIP. London.

Repper, J, M., \& Perkins, R, E. 2001. Working Alongside People with Long Term Mental Health Problems. Stanley Thornes Ltd. Cheltenham.

Repper, J. \& Perkins, R. 2003. Social Inclusion \& Recovery. A Model for Mental Health Practice. Oxford: Elseviere Science.

Romme, M. \& Esher, S. 1993. Accepting Voices. Mind. London.

Rush, B. 2004. Mental Health Service User Involvement in England: Lessons from History. Journal of Psychiatric \& Mental Health Nursing 2004, 11, 313-318.

Sainsbury Centre for Mental Health. 2006. Policy Paper: The Future of Mental Health: A Vision for 2015. Sainsbury Centre for Mental Health. London.

Shepherd, G., Boardman, J. \& Slade, M. 2008. Make Recovery a Reality. Sainsbury Centre for Mental Health. London. 
Social Care Institute of Excellence. 2007. Collection of Examples of Service User \& Carer Participation in Systematic Reviews: Research Resource 02.

Tew. J., Gell. C. \& Foster. S. 2004. Learning from Experience: Involving Service Users \& Carers in Mental Health Education \& Training. Mhhe/NIMHE/Trent NHS.

Trent NHS. 2005. Principles for Practice: Involving Service Users \& Carers in Mental Health Education \& Training. Trent NHS Strategic Health Authority.

www.geneticfutures.com/cracked/info/sheet6.asp. 2007 www.mind.org.uk/information/factsheets 2008

www.southwestyorkshire.nhs.uk. 2008 
